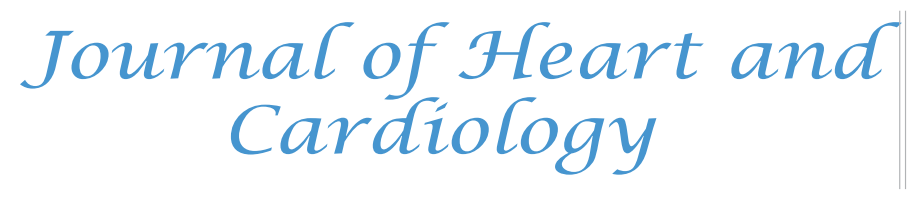

Case Report

OPEN $\boldsymbol{O}$ ACCESS

\title{
Eosinophilic Myocarditis due to Paraneoplastic Syndromes in a Patient with Liposarcoma Recurrence
}

\author{
Wenjuan Bai ${ }^{1}$, Yue Zhong ${ }^{1}$, Hong Tang ${ }^{1}$, Li Rao $^{1 *}$
}

${ }^{1}$ Department of Cardiology, West China Hospital of Sichuan University

*Corresponding author: Li, Rao, M.D., Ph.D. Echo Lab, Department of Cardiology, West China Hospital of Sichuan University, Chengdu 610041, China, Tel: 00862885422355; Mobile: 008618980860406; Email: 1rlz1989@cd120.com

Citation: Li, Rao., et al. Eosinophilic Myocarditis due to Paraneoplastic Syndromes in a Patient with Liposarcoma Recurrence. (2016) J Heart Cardiol 2(1): 28-32.

Received Date: December 23, 2015, Accepted Date: May 04, 2016, Published Date: May 06, 2016

DOI: $10.15436 / 2378-6914.16 .017$

\section{Summary}

Here, we report a rare case of eosinophilic myocarditis due to paraneoplastic syndromes in a patient with liposarcoma recurrence. We suspect that the liposarcoma released cytokines, which resulted in increased eosinophil production and subsequent endomyocardial inflammation.

\section{Case presentation}

A 45-year-old male presented with a 1 month history of cough, fatigue, and dyspnea on exertion, but no fever, chest pain, or hemoptysis. Six years earlier, he was diagnosed with retroperitoneal fibromatosis and underwent resection of the tumour and right kidney.

\section{Investigation}

All his vital signs were normal upon initial presentation. Cardiovascular examination revealed normal heart sounds, with no murmurs. A few moist rales were audible at both lung bases, and edema of the lower extremities was noted. Laboratory analysis revealed the following: white blood cell count, $39.57 \times 10^{9} / \mathrm{L}$ with $39 \%$ eosinophils (reference ranges, $3.5-9.5 \times 10^{9} / \mathrm{L}, 0.4-8.0 \%$ ), and elevated D-dimers, $12.95 \mathrm{mg} / \mathrm{L}$ (reference range, $<0.55 \mathrm{mg} / \mathrm{L}$ ). Tests for myocardial damage were positive: the troponin-T level was $302.9 \mathrm{ng} / \mathrm{L}$ (reference range, $<14 \mathrm{ng} / \mathrm{L}$ ), and the pro-BNP level was $5325 \mathrm{pg} / \mathrm{ml}$ (reference range, $0-277 \mathrm{pg} / \mathrm{ml}$ ). Blood cultures and serologic tests for bacterial and parasitic infections, respectively, were negative. Bone marrow examination revealed hypercellularity and eosinophilia, but no malignant cells. Both contrast-enhanced Computed Tomography (CT) scan and abdomen ultrasound (Figure 1) showed a bulky mass $(10 \mathrm{~cm} \times 11 \mathrm{~cm})$ with inhomogeneous density in the right retroperitoneal area, suggesting invasion of the right liver lobe as well as the inferior vena cava. In addition, intraluminal filling defects were observed at the left pulmonary artery bifurcation, as well as in the segmental pulmonary arteries of the right inferior, left upper, and left inferior lung lobes. However, there was no abnormality detected in the heart structure by CT scan. Then, transthoracic echocardiography was performed, and revealed thickening of the endomyocardium with an irregular margin and high echogenicity (Figure 2A and 2B, Movie I, II). Pulsewave Doppler imaging of mitral in flow demonstrated that the ratio of $\mathrm{E}$ wave to A wave was low, and the deceleration time was

Copyrights: (C) $2016 \mathrm{Li}$, Rao. This is an Open access article distributed under the terms of Creative Commons Attribution 4.0 International License.

Li, Rao., et al. 
long. Tissue Doppler imaging of mitral annulus velocity showed the ratio of e' to a' less than 1 . All these suggested a restrictive left ventricular diastolic filling pattern. Colour Doppler imaging showed there is a moderate tricuspid regurgitation. The chamber sizes and wall thickness were normal, and the LV and RV systolic function was preserved. No thrombus was detected in the endocardium. There is no finding of 12-lead ECG. Based on the evident recurrence of retroperitoneal sarcoma and the unusual echocardiographic images (indicative of profound eosinophilia), a diagnosis of Eosinophilic Myocarditis (EM) due to Paraneoplastic Syndromes (PS) was considered. Heart failure was categorized as New York Heart Association Class II.

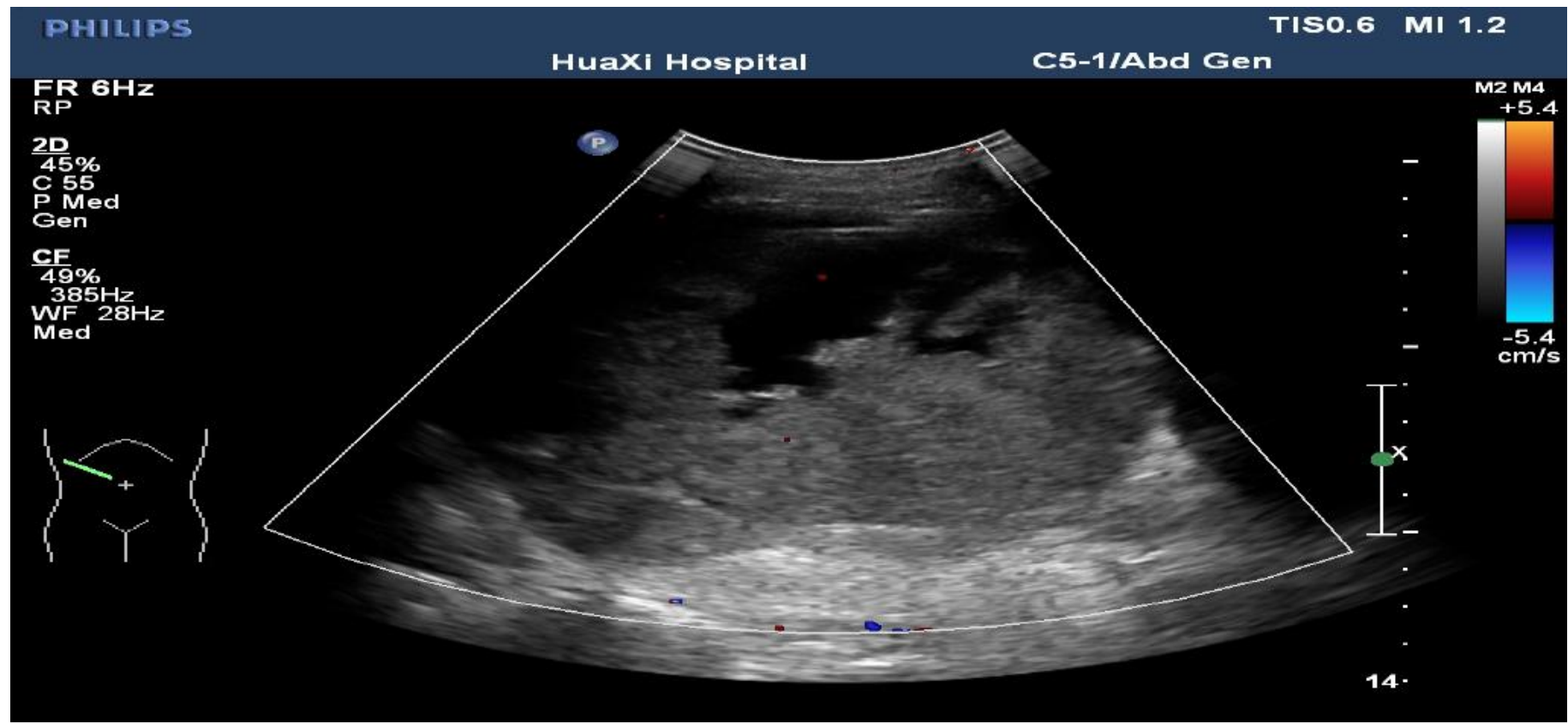

Figure 1: Abdomen ultrasound diplayed a giant intraperitoneal tumor.

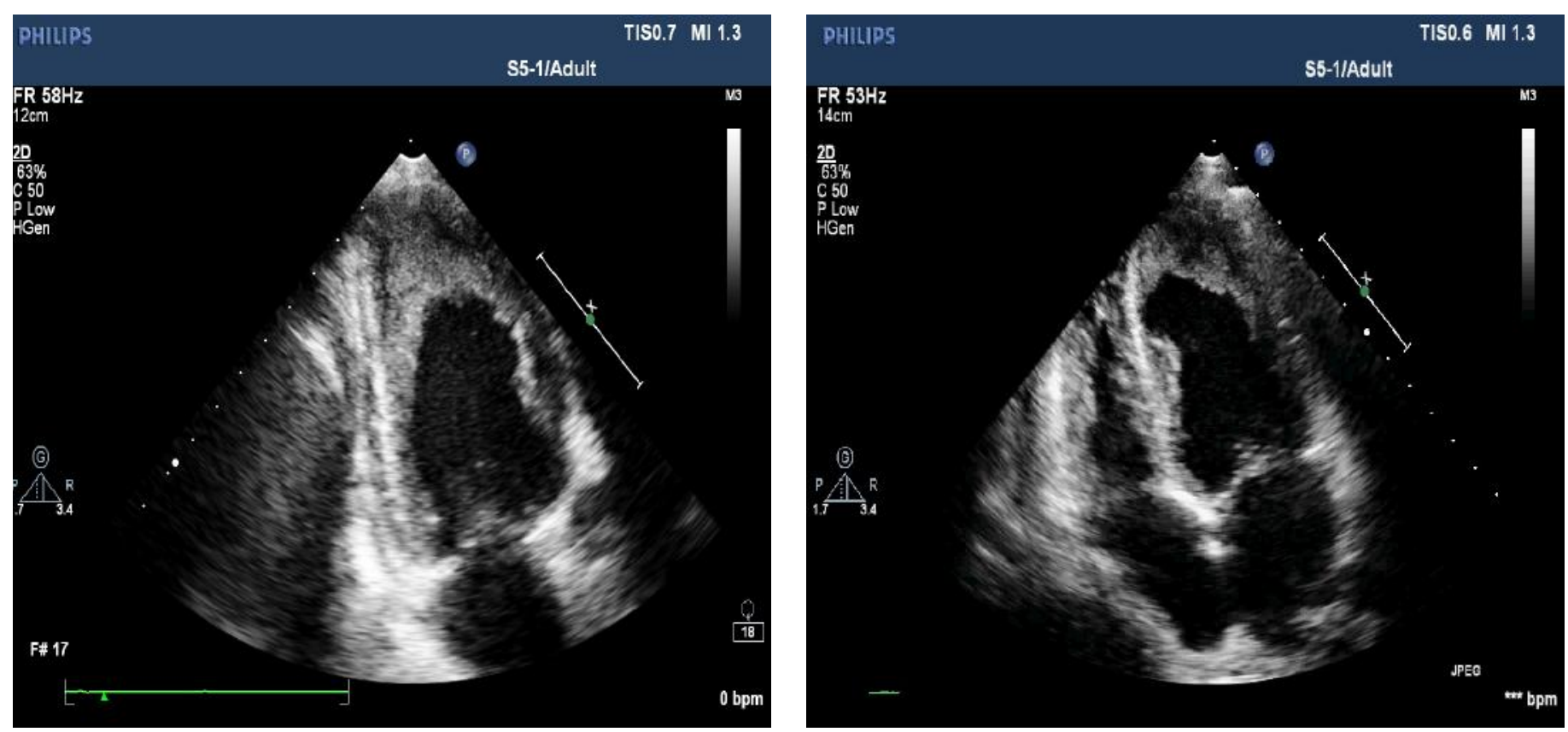

Figure 2: (A) Apical-4-chamber view and (B) apical-2-chamber view of the heart showing thickening of the endomyocardium around the left ventricle. 


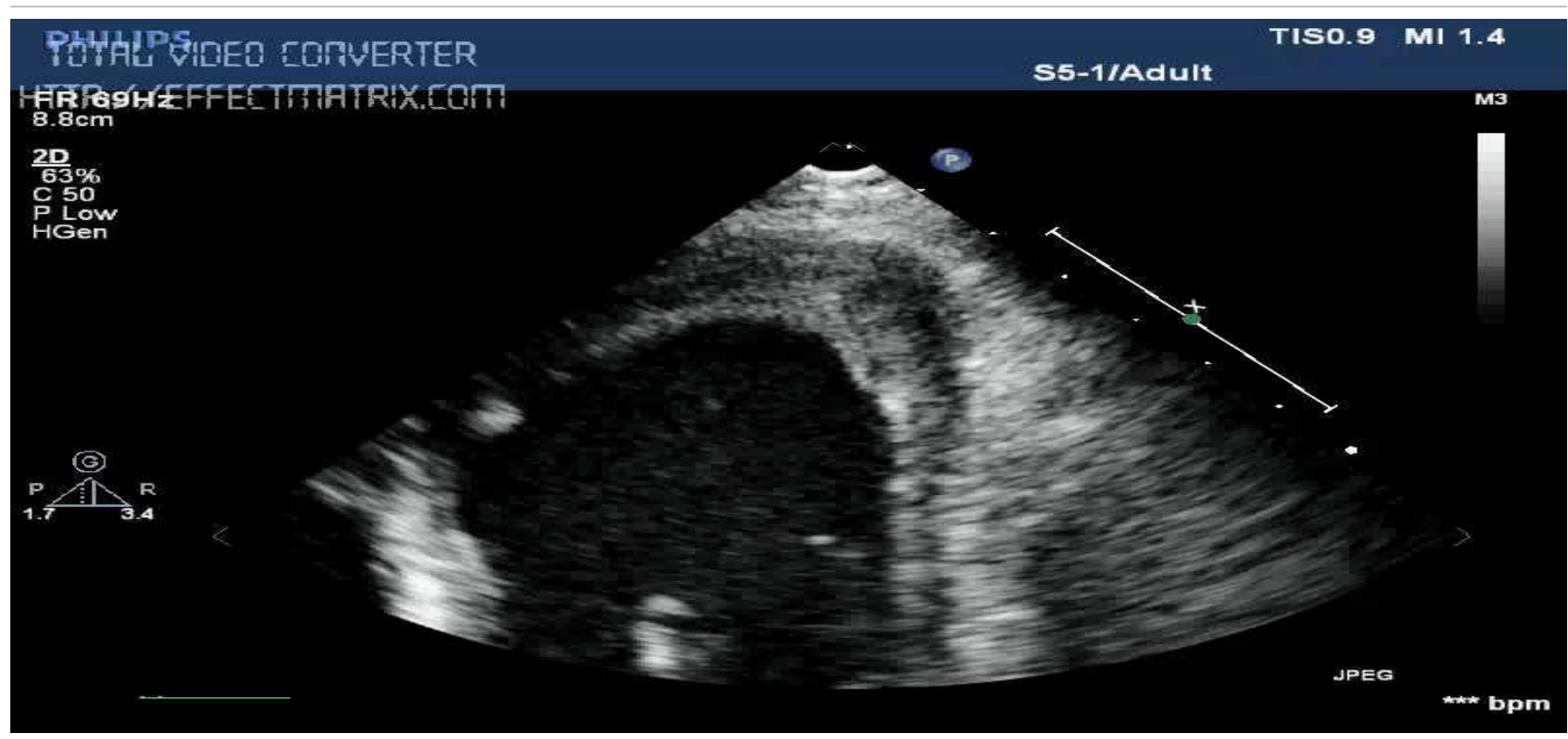

Movie I:

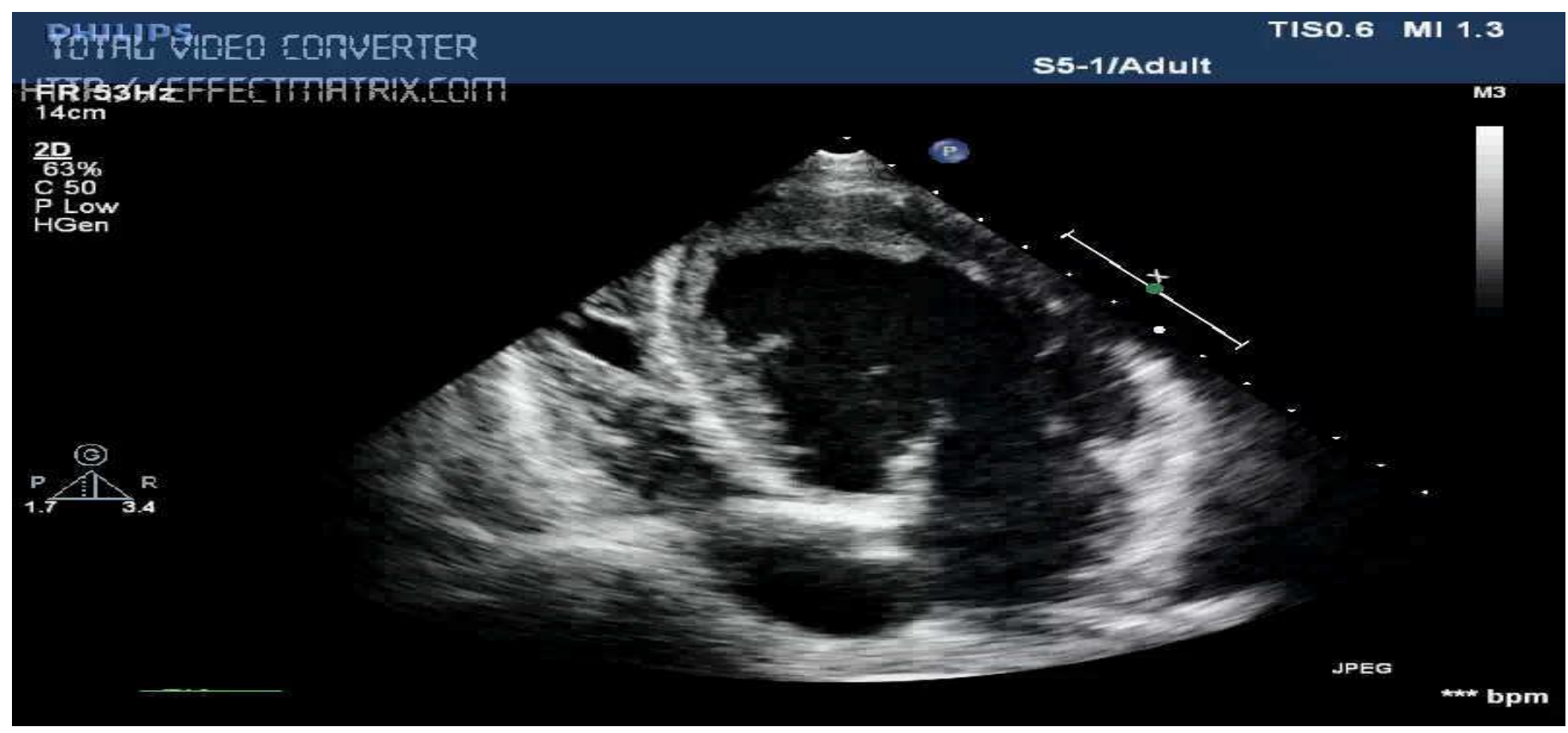

Movie II

\section{Treatment and outcome}

Parenteral anticoagulation was initiated with enoxaparin to treat the suspected pulmonary embolism, and fusemidum was prescribed to treat heart failure. Pulmonary artery angiography was performed 20 days after the initiation of anticoagulation therapy; no abnormalities were detected. Despite this, the patient's white blood cell and eosinophil counts remained high $\left(33.62 \times 10^{9} / \mathrm{L}\right.$ and $28.58 \times 10^{9} / \mathrm{L}$, respectively). For liposarcoma recurrence, no steroid was used during the treatment.

A laparotomy revealed that the mass arose in the retroperitoneum, from where it infiltrated the right lobe of the liver, the inferior vena cava, and the right diaphragm. The patient underwent right hepatectomy and resection of the mass combined with thrombectomy to remove the tumour thrombus from the inferior vena cava. The excised specimen had a hard surface with necrotic lesions inside. The margins (evaluated microscopically) showed evidence of liver metastasis. Histologic examination identified a high-grade dedifferentiated liposarcoma with a high proliferation index (30\% as assessed by Ki-67 immunore activity) (Figure 3A and 3B). Additional immunohisto chemical investigations support a diagnosis of dedifferentiated liposarcoma with liver metastasis. The degree of hypereosinophilia fluctuated throughout the patient's clinical course, first decreasing immediately after debulking surgery and then progressively increasing on postoperative day 2-3, which may suggest residual tumour metastases (Figure 4). 

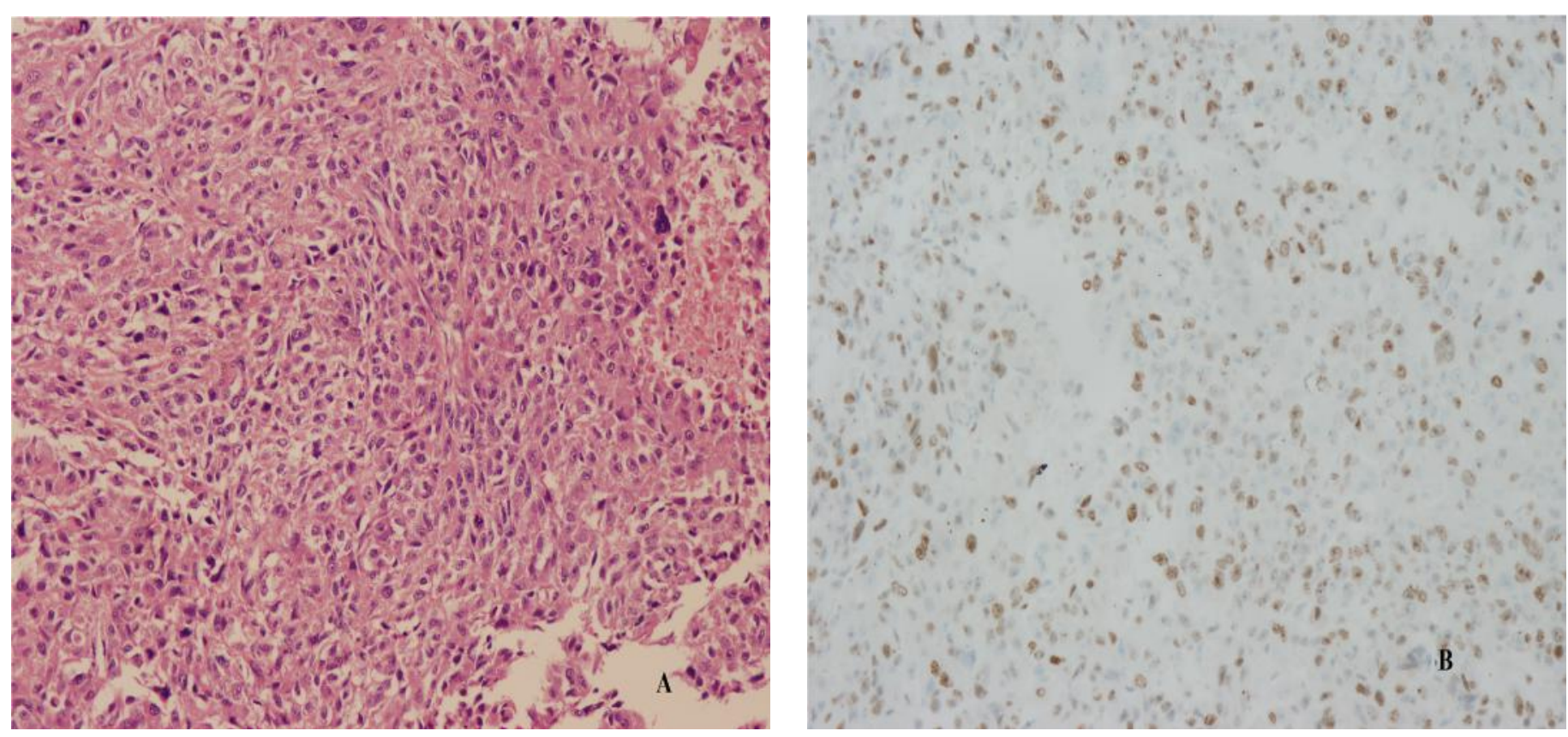

Figure 3: (A) Histologic examination identified a high-grade dedifferentiated liposarcoma. (B) Ki-67 immunoreactivity showed the tumor with a high proliferation index.

\section{Eosinophils count}

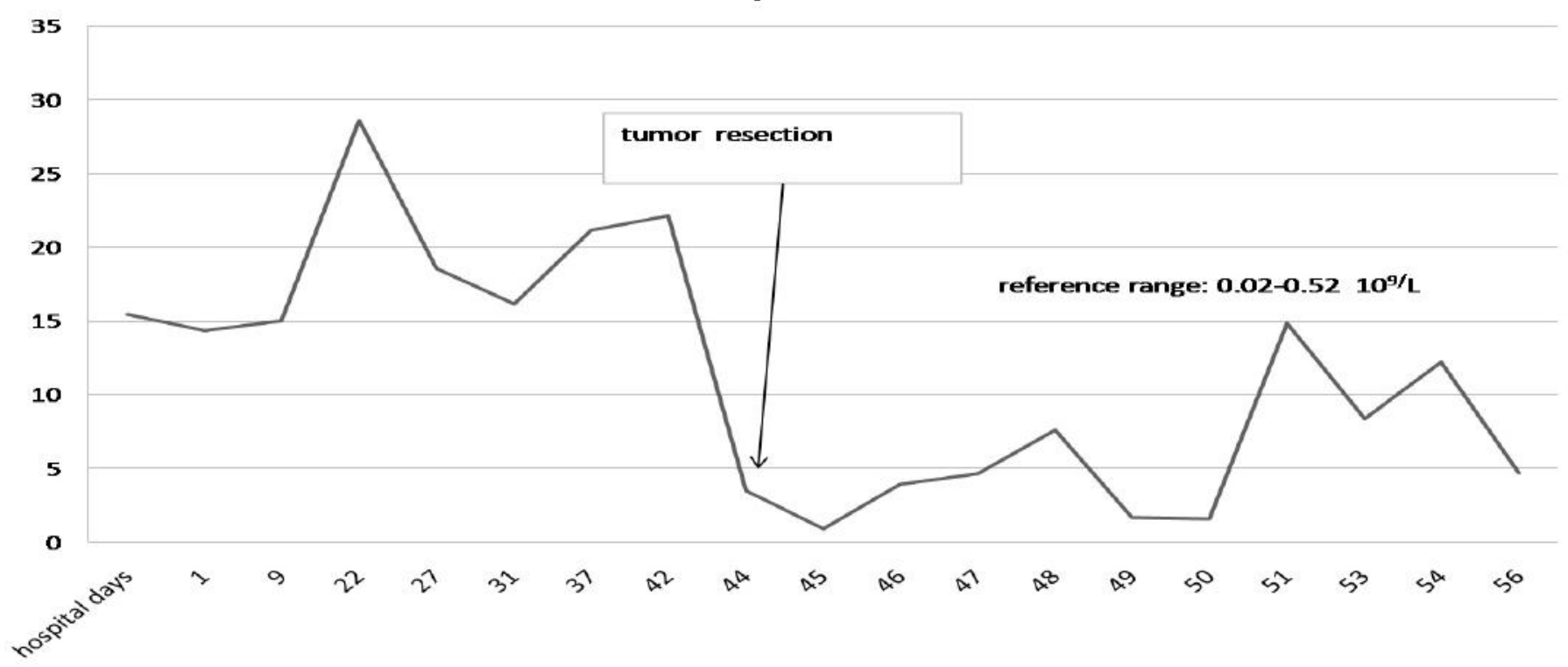

Figure 4: Eosinophil count over time. There was a significant reduction in the count after tumour resection.

In the follow-up, the patient subsequently developed progressive right heart dysfunction and massive pleural effusion and died 30 days after the operation.

\section{Discussion}

Eosinophilic myocarditis is a unique cause of myocarditis. It may be secondary to leukemia, parasite infection, granumatous disease, allergic disease, connective tissue disease, primary hypereosinophilic syndrome, and malignancy ${ }^{[1,2]}$. Eosinophilic myocarditis as a paraneoplastic occurrence is very rare ${ }^{[3,4]}$. The eosinophilia damage to the heart (manifesting as EM) usually proceeds via three common stages: necrosis, thrombosis, and fibrosis ${ }^{[5]}$. Though endomyocardial biopsy remains the gold standard for diagnosing EM, but our hospital cannot do this for historical reason so far. In this case, the echocardiography can provide characteristic images of phenomena strongly associated with this relatively rare disease. For example, unusual and irregular thickening of the ventricular wall is noticeable against a background of increased endomyocardial echogenicity. Furthermore, the most distinctive sign is an obliterative thrombus at the left or right ventricular apex (or both). However, apical contraction remains within the normal range ${ }^{[6]}$. 
Although leucocytosis with liposarcoma is a paraneoplastic phenomenon ${ }^{[7]}$, no case of liposarcoma complicated by PS (which progresses to EM) has been reported. In this case, we speculate that the dedifferentiated liposarcoma secreted cytokines, which then drove the production of eosinophilic granulocytes and their subsequent release from the bone marrow into the peripheral blood. It is suggested that this type of cytokine driven disease progression involve helper cell 2-mediated interleukin-5 production ${ }^{[8]}$. A distinctive feature of the present case is that sustained overproduction of cytokines by the tumour ultimately led to cardiac dysfunction.

The patient reported herein presented with clinical heart failure (although the ejection fraction was preserved) due to restrictive dysfunction of LV, profound eosinophilia caused by cytokines produced by the dedifferentiated liposarcoma, and a typical picture of asymmetrically distributed endomyocardial thickening upon echocardiography, all of which are suggestive of EM. The therapy is usually focused on cardiac support and the underlying etiology of eosinophilia. Usually, the steroid is used to treatment eosinophilic myocarditis, and in this case, for liposarcoma recurrence, no steroid was used. In addition, there was pulmonary involvement in the absence of right ventricular thrombi. A previous study suggests that intraluminal filling defects radiologically-detected in patients with eosinophilia could represent both thrombus formation and the accumulation of eosinophils due to margination in the lung vasculature ${ }^{[9]}$. We believe that the latter occurred in this patient because nothing abnormal was detected upon pulmonary artery angiography.

Conclusion: In conclusion, this case reported herein showed that liposarcoma may cause EM, which manifests with hypereosinophilia.

Funding statement: This research did not receive any specific grant from any funding agency in the public, commercial or not-forprofit sector.

Declaration of interest: There is no conflict of interest needs be disclosure.

\section{References}

1. Tefferi, A., Patnaik, M.M., Pardanani, A. Eosinophilia: secondary, clonal and idiopathic. (2006) Br J Haematol 133(5):468-492.

2. Ommen, S.R., Seward, J.B., Tajik, A.J. Clinical and echocardiographic features of hypereosinophilic syndromes. (2000) Am J Cardiol 86(1):

110-113.

3. Baandrup, U. Eosinophilic myocarditis. (2012) Herz 37: 849-852.

4. Ammirati, E., Stucchi, M., Brambatti, M., et al. Eosinophilic myocarditis: a paraneoplastic event. (2015) Lancet 385(9986): 2546.

5. Lin, D.A., Boyce, J.A. The idiopathic hypereosinophilic syndrome. (2003) Allergy Asthma Proc 24(6): 417-420.

6. Lodha, A., Haran, M., Shetty, V., et al. Hypereosinophilic syndrome presenting with biventricular cardiac thrombi. (2010) Echocardiography. 27(6): E57-E59.

7. Des Guetz, G., Mariani, P., Freneaux, P., et al. Paraneoplastic syndromes in cancer: Case 2. Leucocytosis associated with liposarcoma recurrence: Original presentation of liposarcoma recurrence. (2004) J Clin Oncol 22(11): 2242-2243.

8. Nakamura, A., Tanaka, S., Takayama, H., et al. A mesenteric liposarcoma with production of granulocyte colony-stimulating factor. (1998) Intern Med 37(10): 884-890.

9. Karnak, D., Kayacan, O., Beder, S. Hypereosinophilic syndrome with pulmonary and cardiac involvement in a patient with asthma. (2003)

CMAJ 168(2): 172-175.

Journal ISSN: 2378-6914 (online)

Journal Title: Journal of Heart and Cardiology

Short title : J Heart Cardiol
Ommega Online Publishers

E-mail: cardiology@ommegaonline.com

Website: www.ommegaonline.org 